\title{
DALLA COMPASSIONE ALLA MASSERIZIA: \\ UNA 'CONVERSIONE' DEL MESSAGGIO DI LOTARIO \\ IN QUELLO DI BONO
}

\section{ERIKA PAPAGNI*}

Riassunto: L'intervento si pone l'obiettivo di analizzare i trattati Della miseria delluomo di Bono Giamboni e il De miseria humane conditionis di Lotario de' Segni (Papa Innocenzo III), allo scopo di rilevarne le differenze, ma soprattutto di interpretarle, per capire in che modo la diversa testualità delle due opere corrisponde a due contesti storici profondamente diversi. Il De miseria humane conditionis ebbe, com'è noto, una grande influenza sulla cultura medievale, e fu tradotta e rielaborata in tutte le lingue europee. Poca attenzione però è stata prestata ai volgarizzamenti di quest'opera, in particolare al primo rifacimento italiano, Della miseria dell'uomo, realizzato nella seconda metà del Duecento dal giudice fiorentino Bono Giamboni. Nel messaggio di Bono la scoraggiante analisi della vita terrena che aveva fatto Lotario diviene un trattato morale secondo una mentalità più realistica e serena.

\section{Introduzione}

Vissuto nell'ultimo scorcio del Duecento, il giudice Bono Giamboni rappresenta la chiave del passaggio tra il Medioevo feudale e quello comunale. La sua opera, oltre a voler essere divulgativa e morale, è anche un tentativo riuscito di conferire dignità stilistica e letteraria al "volgare", e si inserisce nello stesso quadro storico-letterario che caratterizza la giovinezza e la preparazione sociale, culturale e politica di Dante Alighieri. ${ }^{1}$ Nelle parole del Debenedetti:

* Questo lavoro è stato presentato al convegno annuale della Canadian Society for Italian Studies (CSIS) in collaborazione con il Dipartimento d'Italianistica dell'Università di Trieste e il Centro per gli Studi Canadesi dell'Università di Udine, tenutosi presso l'Università di Trieste, Italia, 29 maggio - 3 luglio, 2007. Ringrazio Paolo Divizia per le preziose indicazioni sulle fonti secondarie utilizzate da Bono Giamboni. Lo studioso sta preparando l'edizione critica del trattato Della miseria dell'uomo.

${ }^{1}$ Cfr. Cesare Segre, introduzione a Bono Giamboni, Il libro de' Vizi, XXV-XXIX. 
se non si trascurasse tanto, come oggidì suol farsi, la nostra prosa delle origini, per correr dietro a poeti e poetucoli, senza dubbio la vita e le opere di Bono Giamboni sarebbero meglio note. Perché nel quadro della cultura fiorentina, dopo ser Brunetto, conviene proprio ricordar di lui, il nostro Giudice. Traduttore dal latino e dal francese in solenni periodi, apre la schiera dei forti volgarizzatori che difendono mirabilmente il toscano, e preparano l'avvento del Decameron. Uomo di legge e letterato, in Italia, ove il giure e la bella letteratura si sposarono così per tempo, dando così bei frutti, favorisce anch'egli il laicizzarsi della dottrina, il suo divenir popolare ${ }^{2}$.

Bono Giamboni è un giudice fiorentino vissuto nella seconda metà del XIII secolo. Nato verso il 1240 visse fino al 1292 circa. Oltre all'impegno politico e civile nella Curia del Sestiere di Por San Piero, si dedicò con passione all'attività letteraria. Fu uno tra i primi e più grandi volgarizzatori di opere latine del suo tempo: è certamente autore dei volgarizzamenti delle Historiae adversus Paganos di Paolo Orosio, composto su istanza di Lamberto degli Abati e dell'Epitoma rei militaris di Publio Flavio Vegezio Renato, conservato in sei codici, dedicato a Manetto (Manente) della Scala, banchiere e uomo politico fiorentino (Segre 1986). Sono di Bono le prime due redazioni ( $\alpha$ e $\beta$ ) del Fiore di rettorica (Speroni 1994) 3 .

In passato gli sono attribuite anche altre opere, ma la critica più recente ha mostrato false le attribuzioni dei volgarizzamenti del Livres dou trésor di Brunetto Latini (comprese le opere ad esso collegate: volgarizzamento dell'Etica Nicomachea di Aristotele e volgarizzamento del De formula vite honeste di Martino da Braga) e del Giardino di consolazione (Segre 1986)4.

Così Simona Foà sintetizza la figura di Bono Giamboni nel Dizionario biografico degli Italiani:

Esponente di rilievo di quella intellettualità fiorentina che esercitava l'attività giuridica, che partecipava attivamente alla vita politica della città e che, attraverso i volgarizzamenti e la scrittura di opere originali in volgare, era impegnata nella creazione di una nuova cultura laica (Foà, DBI, 304).

Nella Toscana del Duecento, infatti, si diffonde rapidamente una let

2 Debenedetti, "Bono Giamboni”, 271.

3 Bono è stato riconosciuto come autore, e non più come rimaneggiatore, del Fiore di rettorica, dallo Speroni in Bono Giamboni, Fiore di rettorica, a cura di Gian Battista Speroni, Pavia, Dipartimento di Scienza della Letteratura e dell'Arte medioevale e moderna. Redazione $\alpha, 3-107$. Redazione $\beta, 109-127$.

${ }^{4}$ Vol. I (A-Col), 377-379. 
teratura in prosa, come segno della progressiva importanza del volgare, "nella predicazione, nella divulgazione scritta di materie morali e religiose, o di argomenti storici e retorici e di narrazioni laiche, grazie alla domanda del pubblico che si va costituendo nei Comuni toscani" ${ }^{5}$. La sua feconda attività di traduzione gli portò da un lato molta fama, dall'altro offuscò la conoscenza di Bono Giamboni come scrittore originale ed autonomo. È soltanto in tempi recenti che la sua opera originale, il Libro dei Vizi e delle Virtudi, è stata valorizzata.

Fu il primo in Italia ad elaborare una prosa dottrinale, narrativa, didattica ed eloquente col suo Libro de'Vizi e delle Virtudi. Testo fondamentale della prosa volgare predantesca, intitolato dagli Accademici della Crusca Introduzione alle virtù. $\grave{\mathrm{E}}$ conservato in quattordici codici ${ }^{6}$, ed è un trattato di argomento morale in forma allegorica che rispecchia appieno certi procedimenti istituzionali della politica e della società comunale, nonché la morale laica di Bono. Ma l'opera a parer mio più interessante, per diversi motivi che vedremo in seguito, anteriore al Libro de' Vizi e delle Virtudi, è senza dubbio il trattato di carattere morale Della miseria dell'uomo di cui non esiste ancora un'edizione critica. La mia analisi è stata condotta quindi sull'edizione data alle stampe da Francesco Tassi a Firenze nel 1836. Fonte principale del Della miseria dell'uomo è senz'altro il trattato di carattere religioso De contemptu mundi sive de miseria humane conditionis di Lotario Diacono (poi divenuto Papa Innocenzo III), scritto nel 1191, che ebbe nel Medioevo un'immensa diffusione; Bono ha però inserito interi capitoli tratti da altri autori, e soprattutto ha trasformato completamente lo spirito del trattato di Lotario. Utilizza senza economia numerosi testi latini, tra cui in particolare il De amore et dilectione Dei di Albertano da Brescia7. Scopo di questo saggio è di mostrare in che modo Bono Giamboni, che pure riprende 'pari pari' gli argomenti di Lotario, riesce però a trasformarne la portata e a darci così il senso del nuovo atteggiamento verso la vita, quella terrena e quella ultraterrena, che si diffonde nella cultura comunale italiana.

5 Barberi Squarotti, Storia della civiltà letteraria italiana, 1:338

6 Paolo Divizia, Ancora un compendio del Libro de' Vizi e delle Virtudi di Bono Giamboni, "Medioevo Romanzo", XXVII/1 (2003), 33, nota 1. Il quindicesimo manoscritto, Padova, Biblioteca Civica, A.18, è stato segnalato successivamente da A. Mazzon, Il "Libro de’ Vizi e delle Virtudi" di Bono Giamboni. Un nuovo testimone presso la Biblioteca Civica di Padova, "Critica del Testo", iv/2 2001, pp. 461-67.

7 Segre, Bono Giamboni, in Dizionario critico della letteratura italiana, 1:382. 


\section{Bono e Lotario a confronto sulla 'miseria della condizione umana'}

Il De contemptu mundi sive de miseria humane conditionis è diviso in tre libri secondo l'evoluzione dell'età (nascita, vita adulta, morte) e come enunciato nel prologo Lotario vuole mostrare la bassezza della condizione umana e afferma che per farlo deve umiliare la superbia la quale è "il capo di tutti i vizi" (l'origine di tutti i vizi). L'uomo è stato generato nel peccato, è nato per la pena, il timore e il dolore, la sua vita è fatica e, ciò che è più miserevole, è nato per la morte; in più, l'essere umano commette "azioni vane" per cui trascura ciò che è serio, utile e necessario. Lo spirito che regna sull'opera è basato sulla negazione della vita terrena e delle vanità. Il messaggio implicito che si ricava dall'opera di Lotario è che l'unica vita giusta è quella monacale, proprio perché rappresenta la rinuncia al mondo e il riconoscimento che l'unica salvezza possibile è in Dio.

Il trattato morale Della miseria dell'uomo di Bono Giamboni è suddiviso in un prologo e otto trattati (preceduti da un capitolo introduttivo che dichiara il "programma della trattazione"), che illustrano (mantenendo la tripartizione di Lotario: (1) la miseria dell'uomo e della femmina dall'ora che è creata fino alla nascita, (2) la miseria dalla nascita alla morte, a causa del dolore, della fatica, della paura, della morte, (3) la miseria dopo la morte: pene dell'Inferno, ma anche la beatitudine e la gloria del giusto, ed il Giorno del giudizio. Ispirato alla scena iniziale del De consolatione philosophiae di Boezio, il prologo annuncia che Bono si propone (a) "di consolare coloro, che delle tribolazioni del mondo si sentono gravati", (b) di invitare "coloro che sono rei, [a] umiliarsi e convertirsi, considerando il loro malvagio stato e pessima condizione, a che sono dati in questo mondo e nell'altro," ma anche (c) di dare "conforto e vigore a coloro, che sono buoni, di migliorare, per la speranza che mostra del loro guidardone". Lo spirito che regna sull'opera è retto da una prospettiva che volge verso un punto di fuga positivo, Bono vuole consolare e confortare chi legge e dare una speranza di salvezza sia in questa vita che dopo la morte. La mentalità di Bono lo porta a cercare delle soluzioni pratiche ai problemi che si erano instaurati all'interno del comune e, sotto questo punto di vista la sua opera diventa un vero e proprio manuale per vivere sereni.

Raggruppo gli argomenti principali dell'opera di Lotario delineandone la struttura logica: miserie della vita nella sua corporeità fin dalla concezione; miserie della corporeità dell'età adulta; le fatiche dei mortali; le diverse miserie secondo lo stato sociale; i nemici dell'uomo che fanno della vita un perpetuo combattimento; il corpo come carcere dell'anima; lo spavento dei sogni; dolori dovuti alla compassione; gli infortuni inaspettati, gli 
scherzi della sorte; la distorsione totale dell'umano giudizio; il desiderio delle ricchezze che porta a due vizi: la cupidigia e l'avarizia; la ricerca dei piaceri che porta a tre vizi: gola, ubriachezza e lussuria; la ricerca degli onori e del potere che fa nascere: ambizione, superbia e arroganza; la disordinata concupiscenza degli ambiziosi; l'arroganza degli uomini; il momento della morte; i quattro dolori che i cattivi patiscono nella morte; le quattro venute di Cristo sulla terra ${ }^{8}$; le diverse pene dell'inferno; il giorno del giudizio.

Corrispondentemente, gli argomenti di Bono si raggruppano secondo la seguente struttura logica: miseria dell'uomo $e$ della femmina dall'ora che è creata fino all'uscita del ventre della madre; miseria dalla nascita alla morte: dolore-fatica-paura; i rimedi; le fatiche: per divenire savio delle cose, per radunare ricchezze, per i desideri della carne, per conseguire signorie e onori; il vizio della " cupiditade " e il vizio dell'avarizia; i rimedi; che cosa fa buona la ricchezza (la cortesia); dai desideri della carne nascono due vizi: il vizio della gola ed il vizio della lussuria; i rimedi contro la lussuria; dal desiderio delle signorie e degli onori nascono due vizi: superbia e vanagloria; le quattro paure: demonio, carne, uomo, natura; sogni; i rimedi; la morte; la miseria dopo la morte; quali sono i comandamenti di Dio, che devono essere osservati per non andare all'inferno; la sorte dell'anima dopo la morte: l'inferno e i suoi tormenti; la beatitudine e gloria del giusto che va in Paradiso; come è disposto il Paradiso e come le potenze dell'anima (immaginare, lavorare e desiderare) trovano compimento e soddisfazione in Paradiso; il giorno del Giudizio?.

Come si può vedere dalle descrizioni degli argomenti principali delle due opere, la differenza più importante tra i due testi sta nel fatto che a tutte le fatiche e le miserie riprese dall'opera di Lotario, Bono contrappone dei rimedi: rimedi contro il dolore, la fatica e la paura; rimedi contro i vizi; rimedi contro le quattro paure; e nel fatto che alla descrizione dell'inferno Bono giustappone la visione del Paradiso, indicando anche i comandamenti, che devono essere osservati per meritare il Paradiso. Insomma per Bono la vita è fatica ma tutti troveranno il meritato riposo e si sazieranno in Paradiso. La prima volta in cui Bono parla dei rimedi, egli afferma che i mali si possono normalmente evitare con la previdenza, e aggiunge che, se poi i mali non si potessero evitare, la pazienza stessa che è necessaria per

8 Questo capitolo compare nell'edizione Maccarrone ma non si legge nell'edizione Lewis.

9 Il capitolo sui quindici segni del Giudizio che si legge nell'edizione Tassi è spurio, vd. Divizia, I quindici segni del Giudizio, 47-64. 
sopportarli porta comunque con sé alcuni beni : infatti, chi sopporta le tribolazioni di questo mondo in pace ha il vantaggio di "compiacere Dio" (primo bene: II, 4); si rende simile a Dio e ai santi e questa è "la via de' buoni, non vuole essere buono chi delle tribolazioni del mondo non vuole sentire"10 (secondo bene : II, 5); guadagna il paradiso - e Bono cita l'Apostolo: "Se noi siamo compagni di Dio nelle passioni, sì saremo suoi compagni nelle consolazioni"11 (terzo bene: II, 6).

Va notato inoltre che fin dal primo trattato Bono mette l'enfasi su una questione a lui cara: si inizia a parlare della femmina. Con la "miseria dell'uomo e della femmina dall'ora che è creata fino alla nascita," la donna viene inclusa in questa opera; mentre nell'opera di Lotario non se ne parla se non in modo negativo probabilmente perché, nella sua visione, la donna è semplicemente fonte del peccato.

La seconda volta che Bono parla dei rimedi è nell'undicesimo capitolo del terzo trattato (le "fatiche") in cui inizia a parlare della questione delle ricchezze. Bono dedica molti più capitoli a questo argomento rispetto a Lotario ed inoltre affronta il discorso in modo diverso. Insiste sul fatto che ci sono modi per usare le ricchezze in modo corretto; rivela comunque il suo interesse per la salvezza eterna quando conclude che la povertà è migliore della ricchezza e mette di nuovo l'accento sulla virtù della pazienza (di cui Lotario non parla). Bono indica dunque che cosa fa buona la ricchezza (III, 13): non bisogna desiderare di volere troppo, ma ciò che basta per vivere bene. Spiega come si devono guadagnare le ricchezze (III, 13), come si devono spendere e usare (III, 14), come si devono conservare (III, 15) e quali sono le altre cose che fanno buona la ricchezza (III, 16) come ad esempio la cortesia ovvero in che modo l'uomo ricco deve essere cortese e come deve usare la cortesia: deve frenare la lingua, deve temperare il cuore, deve spendere e donare le sue ricchezze in base alle sue possibilità (III, 17). Dà spazio all'opinione secondo cui le ricchezze sono preferibili alla povertà (III, 11), ma afferma poi che anche la povertà può essere buona (III, 12); e spiega perché la vita povera viene considerata dai Santi come beata, perfetta e migliore di quella ricca (III, 18).

Lotario, invece, dedica molti più capitoli alla condanna dei vizi della cupidigia e dell'avarizia e al contrario di Bono mette l'accento sull'influenza delle ricchezze nell'amministrare la giustizia. Insiste sulla corruzione dei giudici che accettano gli "iniqui doni” e che non vivono per la giustizia, ma per il denaro, parla "della vendita della giustizia": i (giudici) ricchi sono

\footnotetext{
10 Giamboni, Della miseria dell'uomo (1836), 31.

11 Giamboni, Della miseria dell'uomo (1836), 33.
} 
corrotti (putrefatti) per amor del denaro e finiscono per imprigionar la loro anima. $\dot{E}$ interessante notare che Bono, che pure è un giudice, non parla affatto di questa questione. Lotario dedica poi gran parte della sua opera ai vizi che nascono dalla ricerca del piacere (gola, ubriachezza e lussuria) e ai vizi che nascono dalla ricerca degli onori: ambizione, superbia e arroganza. Bono ripete le condanne di Lotario, ma preferisce "trovare i rimedi ai vizi".

La terza volta che Bono parla dei rimedi è nel ventunesimo capitolo: "De' rimedj che sono trovati, che l'uomo dee usare contro al vizio della lussuria"12. Fra i tanti vizi generati dai desideri degli uomini, Bono si sofferma soprattutto su questo, forse proprio perché la lussuria è "vizio naturale dell'uomo e della femmina," e i suoi interessi laici lo portano verso l'accettazione della vita terrena e alle possibili soluzioni dei suoi problemi morali. Bono cita Orazio (Epistole 1, 10, 24) per sostenere che essendo un vizio naturale non vi è completamente rimedio: "la natura discaccerai dalla forca, ma ella sempre vi ritornerà" e "né la morte, né l'amore non si può fuggire". Nessuno quindi può scampare alla lussuria. Ma alla fine si persuade che ci sono due rimedi possibili: il matrimonio per coloro che non vogliono essere casti, "per discacciare le tentazioni del nimico, soddisfacendo alla natura"13 e la fuga "per coloro che casti vogliono stare".

La quarta volta che Bono parla dei rimedi è nel secondo capitolo del quarto trattato: "De' rimedj che debbono pigliare le genti sopra le paure". Come detto in precedenza le paure principali sono quattro: demonio, carne, uomo, natura; e secondariamente i sogni: "l'uomo e la femmina sempre debbono stare ammannati per difendersi da loro"14. Ritorna a parlare della pazienza, unica virtù capace di salvare chiunque, e ricorda che spesso i giusti sono tormentati ed i peccatori esaltati: "l'uno commette il peccato e l'altro ne porta la pena; Cristo iusto è crocifisso e Barabas ladro è liberato" 15 . Il giusto sarà sempre perseguitato e disprezzato dal mondo è per questo che deve avere molta più pazienza affinché possa portare in pace le pene del peccatore ma infine meriterà la pace eterna, il Paradiso.

La quinta e ultima volta che parla dei rimedi è nel secondo capitolo del sesto trattato: "Qui si dice delle due comandamenta maggiori, le quali sono principali e capo delle altre". Il primo è "Ama Iddio Signore tuo di tutto il cuore tuo, e di tutta l'anima tua, e di tutte le forze tue" e il secon-

12 Giamboni, Della miseria dell'uomo (1836), 83.

13 Giamboni, Della miseria dell'uomo (1836), 83-84.

14 Giamboni, Della miseria dell'uomo (1836), 97.

15 Giamboni, Della miseria dell'uomo (1836), 98.

16 Giamboni, Della miseria dell'uomo (1836), 109. 
do è "Ama il prossimo tuo siccome te medesimo"16. Bono sostiene che bisogna rispettare i due comandamenti principali, tutti gli altri nascono da questi. Anche qui notiamo che quando parla degli uomini c'è sempre la presenza della donna: "l'uomo e la femmina, per lo comandamento primario, sia tenuto ad amare Iddio [...] e per lo comandamento secondo sia tenuto ad amare il prossimo"17. Bono trova insomma un rimedio ad ogni problema, cerca una speranza, una via d'uscita all'inevitabile condanna lotariana. Passiamo in un certo senso dall'Inferno al Paradiso.

Per dare un esempio concreto di come uno stesso argomento è affrontato in maniera diversa dai due autori, prendiamo il passo che mette a fuoco la condizione del povero e del ricco (III, 11). Questo è un capitolo importantissimo per capire la sensibilità di Bono, e soprattutto ci fa avvertire il diverso contesto storico nel quale le due opere si collocano. Lotario viveva, evidentemente, in una società feudale e Bono in una società comunale. Quando Bono parla della miseria del ricco e del povero, si riferisce alle classi sociali all'interno del comune fiorentino, dove la stragrande maggioranza della popolazione era formata da mercanti, artigiani, da persone che dovevano produrre per vivere. Le parole di Lotario "culpa domini, servi pena" (Cap. XV, I libro) ovvero "se il ricco commette il peccato, il povero ne porta la pena" vengono sentite e descritte da Bono in maniera molto più realistica: "[i poveri] sono molti di servigi richiesti e di fazioni gravati [...] e sì ne sono straziati e sono ingiurati e battuti, e niuno se ne duole"18. Si faccia inoltre attenzione al modo in cui Bono introduce la descrizione delle miserie del povero:

furono certi, che dissono: Pogniamo che le ricchezze siano ree; io ti vo' mostrare che la povertà è vie peggiore, però voglio fuggire povertade e abbracciare ricchezze, perché coloro, che sono poveri d'avere, di manicare e di bere, ecc.

Il giudizio negativo sulla povertà non è di Bono ma è l'opinione di "certi" (va notato che Bono non cita mai Lotario). Inoltre agli argomenti ripresi da Lotario Bono ne contrappone altri:

a rispondere alle cose, che sono dette di sopra, e acciò che possiamo vedere certi ammonimenti, che pongono i Savi sopra la povertade, [...] perché la povertade e la ricchezza può essere buona e rea, sì ti voglio in prima mostrare, che cose debbono essere nel povero, acciò che sia buona la sua

17 Giamboni, Della miseria dell'uomo (1836), 110.

18 Della miseria dell'uomo, Trattato III, cap. 11, (p. 57). 
povertade; appresso che cose debbono essere nel ricco, acciò che sia buona la sua ricchezza. Appresso ti mostrerò come la vita povera è migliore che la ricca, perché ne mena al buono fine con minore rischio, e per più piana via.

Se Lotario insiste sull'inevitabile miseria di qualunque condizione, quella del povero e anche quella del ricco, Bono invece si premura di mostrare come ognuna di queste condizioni può essere 'buona' e quindi non solo sopportabile in questa vita, ma anche meritevole della salvezza eterna. Bono si trasforma in una sorta di educatore. Prende per mano i lettori-destinatari della sua opera e li conduce attraverso un viaggio immaginario, adoperando un linguaggio amichevole, e quando incontrano la "miseria del povero e del ricco", Bono avverte prima di tutto, appoggiandosi all'autorità Boezio, che: "Neuna cosa è misera all'uomo, se non in quanto egli pensa che misera sia." I suoi lettori vedono così che la povertà può essere una cosa positiva, specialmente da un punto di vista morale, e affinché lo sia bisogna osservare determinate condizioni (Trattato III, Cap. 12).

La visione del mondo di Bono differisce completamente da quella di Lotario. In Lotario ogni essere umano è condannato all'inevitabile fatica, va ricordata la sua affermazione: "meglio morire nel ventre della madre piuttosto che nascere". Lotario è prima di tutto un monaco, ed ha quindi rinunciato alla vita terrena: infatti, come membro di un ordine religioso, il monaco ha rinunciato al mondo, pronunciando i voti solenni di povertà, castità e obbedienza. L'unica scelta di vita accettabile agli occhi di Lotario è la sua: quella monacale. Entrambi si rivolgono ai laici, ma Lotario lo fa soltanto per ammonirli e per concludere che non "valgono nulla" e che l'unica strada di salvezza possibile è la rinuncia al mondo terreno, la vita monacale e quindi Dio. Il messaggio di Bono è l'anti De contemptue il suo motto potrebbe essere: "la vita è fatica ma esistono i rimedi!". Le parole di Bono confortano chi legge, guidano ogni lettore, qualsiasi sia il loro stato sociale, a trovare il modo di vivere bene in questa vita e a meritare il Paradiso dopo la morte. Con Lotario assistiamo alla svalutazione assoluta di tutto quello che è terreno; egli si colloca al di sopra dei comuni mortali, si eleva al di sopra dei laici, si sente in uno stato superiore e separato dal mondo, avendo rinunciato totalmente al mondo. Tutti gli esseri umani devono riconoscere la vanità della vita terrena per potersi salvare, proprio come fanno i monaci. Con Bono, invece, assistiamo alla rivalutazione di quello che è terreno, egli fa parte di questo mondo, non si eleva al di sopra degli altri, anzi riconosce di far parte del pubblico al quale rivolge la sua opera. Scrive per consolarsi e per consolare: trova i rimedi per vivere bene in questo mondo e guadagnarsi il Paradiso nell'altro. Cerca quindi la sal- 
vezza nella vita terrena non al di fuori di essa.

Altra profonda differenza tra i due testi è lo stile. Lotario procede per brevi citazioni, parla tramite affermazioni autorevoli e infatti la miseria della condizione umana trova la sua auctorictas nei testi delle sacre Scritture: fra i più citati sono i salmi, spesso parafrasati, i libri sapienziali, il libro di Giobbe e quelli dei profeti dell'Antico Testamento, il Vangelo di Matteo e di Luca del Nuovo Testamento. Il suo trattato è una vera e propria guida alla riflessione sulla miseria della condizione umana: onde viene superbia a te, uomo, che il tuo ingeneramento è peccato, il tuo nascere è pena, la tua vita è fati$\mathrm{ca}$, e fa pure bisogno che tu muoia? (la frase rende il senso del Cap. I del libro III del De Miseria). Lo stile di Bono, al contrario, è colloquiale e amichevole, il suo discorso guida i lettori attraverso un ragionamento, per trovare insieme a loro una o più soluzioni ai problemi che devono affrontare nella vita terrena. Possiamo dire che Bono quindi parla alla razionalità dell'uomo: non solo vuole convincere i suoi lettori ma li fa partecipare al ragionamento per arrivare infine alle stesse conclusioni: esistono i rimedi a tutti i problemi quotidiani ma soprattutto non bisogna mai perdersi d'animo perché la vita terrena ci permette di conquistare il Paradiso.

\section{Il contesto}

Per spiegare la trasformazione radicale subita dal trattato di Lotario, si dovrà considerare che Bono è vissuto nel periodo in cui a Firenze si affermava una nuova classe sociale: la borghesia. È proprio nel corso del Duecento che si diffonde una nuova mentalità, più aperta e più terrena, e allo stesso tempo fiorisce una nuova cultura laica che per esprimersi sceglie il volgare al posto del latino. La nuova cultura comunale trasforma e laicizza il contenuto delle opere: ciò che per lungo tempo era stato patrocinio esclusivo della Chiesa, ora fa parte degli interessi mondani e quindi delle nuove esigenze di una società borghese e mercantile. Bono sentiva chiaramente i problemi che si erano creati a causa dei profondi contrasti della società e della vita politica comunale.

Nell'opera di Lotario ci troviamo all'interno della società feudale che, con la sua salda organizzazione gerarchica, riceveva generalmente il consenso dei propri membri, anche di coloro che ne formavano la stragrande maggioranza e pagavano il costo umano più alto ( $\mathrm{mi}$ riferisco ovviamente alla popolazione contadina): probabilmente il feudalesimo offriva un modello sociale ordinato, almeno nella teoria, dotato di un senso e di una scala di valori 19 .

19 Barberi Squarotti. Storia della civiltà letteraria italiana, I:70. 
Con Bono invece siamo all'interno di un'organizzazione comunale:

meno restrittiva nella base del reclutamento, che si apre ad esponenti della nobiltà come ai membri della borghesia mercantile, e controllata da una minoranza strutturata in modo orizzontale, [e] non era esente a sua volta da inconvenienti assai gravi. Se i costi del feudalesimo vengono generalmente accettati dalla coscienza dell'epoca, non altrettanto avviene con le profonde disfunzioni della vita comunale. La lotta delle fazioni, la successione degli esili e delle confische imposte dalla parte di volta in volta prevalente, sono all'origine di un contrasto molto forte tra l'interesse e il bene generale, espresso dalla denominazione stessa di comune, e la spinta alla prevaricazione che sorge dalle parti in lotta fra loro ${ }^{20}$.

Leggendo queste due citazioni, la situazione appare pienamente delineata: Bono, che vive nell'Italia dei Comuni, sente un bisogno di pacificazione, di solidarietà verso il prossimo, è per questo che nella sua opera adotta un atteggiamento cordiale e aperto a tutte le classi sociali.

Al contrario di Lotario, che porta con sé un messaggio prettamente negativo, Bono che in cuor suo cerca un progetto di vita civile per l'Italia comunale, ci trasmette un messaggio totalmente positivo. Inizia il prologo dicendo che vuole dare "conoscimento perché si possano consolare coloro che delle tribolazioni del mondo si sentono gravati", e qui vediamo già la distanza della posizione di Bono da quella di Lotario.

Leggendo Della miseria dell'uomo i lettori vengono avvertiti delle conseguenze negative che le loro azioni potranno avere e di come difendersi dai pericoli che assediano la vita degli uomini, quella terrena e soprattutto quella dopo la morte. Lo scopo principale che Bono si propone è quello di dare a chi legge dei consigli morali per vivere bene in questa vita e per salvarsi in base alla propria posizione sociale. Va ricordato che nel 1250 a Firenze si instaura il Comune del Popolo, ci troviamo in un clima di mobilità sociale all'interno di un Comune che funge da città-stato e con una politica autonoma: una realtà in movimento con valori ben diversi da quelli della statica società feudale. La classe che finisce con l'avere l'egemonia nella società comunale (il popolo-borghesia) valorizza il successo, lo spirito d'iniziativa, l'intelligenza volta alla realizzazione di un progetto, l'accumulazione, il "far masserizia”. Il mercante si sostituisce al guerriero; i commerci, gli scambi, la gestione della cosa pubblica esigono nuove competenze, una figura di intellettuale diversa dal "chierico", una modifica delle strutture scolastiche. Esigenze di rinnovamento e di cambiamento che trovano espressione nel trattato di Bono nonostante la falsariga del modello lotariano.

20 Barberi Squarotti. Storia della civiltà letteraria italiana, 1:70. 
È sbagliato affermare che durante il medioevo si sia ignorato l'uomo: basti pensare al valore che si dà a lui e al suo lavoro nell'età dei comuni. Larte e la letteratura sono espressione di un determinato periodo storico, con tutti i molteplici motivi che lo hanno formato e che sono irripetibili; essa stessa perciò è irripetibile. Né è possibile, d'altra parte, supporre di potersi riallacciare a un'età ignorando gli immediati predecessori: ognuno di noi è quello che è perché nasce e si forma in un certo momento storico. Non si può capire l'atteggiamento di Bono se non si conosce il disprezzo del mondo di Lotario. E tuttavia l'opera di Bono è così diversa perché è diversa la situazione culturale. Egli parla direttamente ai lettori, partecipa e comunica utilizzando la prima persona plurale, e punta ad un pubblico di persone che come lui si avventurano nella nuova vita comunale, che cercano delle risposte nella saggezza della letteratura gnomica, come una sorta di guida al successo o a come evitare l'insuccesso ed i pericoli incombenti della vita quotidiana nel fine di trovare la salvezza eterna qualunque sia la loro condizione sociale. Con il mosaico di citazioni nell'opera di Lotario vediamo che tutti sono miserevoli, tranne che i monaci naturalmente: è visibile che Lotario, al contrario di Bono, non sente la sofferenza dei poveri e ne rimane estraneo e distante. Papa Innocenzo III sosteneva che la vita terrena non è che miseria dal momento del concepimento fino alla morte, e questo senza distinzione di classe sociale, e che tutti gli uomini che desiderano le ricchezze, i piaceri, e gli onori, cadranno inevitabilmente nel peccato e saranno puniti nei secoli dei secoli. Bono, invece, cerca di auto-convincersi e di convincere i suoi lettori che tutti possono salvarsi, tutti possono raggiungere la salvezza eterna nel mondo. E che "chi sopra tutte le avversitadi che gl'incontrano nel mondo vorrà pensare, non sentirà mai bene che sia, perché questo mondo non è altro che miseria [...] ma quelli sono meno tormentati, che per pazienza sanno le cose passare e comportare; perciò che la pazienza [...] tutte le avversitadi vince."

UNIVERSITY OF TORONTO

\author{
APPENDICE I \\ Schema dell'opera di Lotario \\ De contemptu mundi sive de miseria humane conditionis
}

Prologus. Lotario si propone di mostrare in questa opera la bassezza della condizione umana e, afferma che lo fa per umiliare la superbia la quale è "il capo di tutti i vizi (l'origine di tutti i vizi)" e in seguito "la dignità della natura umana col favore di Cristo, affinché il superbo sia umiliato e l'umile esaltato". L'uomo è stato generato nel peccato, è nato per la pena, il timore e il dolore, la sua vita è fatica e, 
ciò che è più miserevole, è nato per la morte; in più, l'essere umano commette "azioni vane" per cui trascura ciò che è serio, utile e necessario.

1. Liber Primus: De miserabili humane conditionis ingressu (lo sventurato ingresso nella condizione umana). Sofferenze della vita fin dalla nascita e senza distinzioni di condizione, classi o età.

\section{Miserie della corporeità dalla concezione fino alla nascita (capp. I-VI)}

I. De miserabili humane conditionis ingressu

"Felici quelli che muoiono prima di nascere, che conoscono la morte prima di conoscere la vita" Geremia $(20,14-18)$ e III cap. di Giobbe.

II. De vilitate materie de qua formatus est homo. (Della viltà della materia di cui l'uomo è formato).

III. De conceptione infantis. (Peccaminosità della concezione).

a. La sofferenza del neonato che viene al mondo e alla vita, già preda del peccato ancora prima di peccare e dell'errore ancor prima di errare;

b. Sin dal concepimento ereditiamo una duplice colpa: la prima sta nel peccato che accompagna l'emissione del seme e la seconda ci viene trasmessa;

c. I nostri genitori ci hanno dato la vita mentre erano avvolti dal peccato.

IV. Quali cibo conceptus nutriatur in utero. (Di qual cibo l'uomo si nutre nel ventre: di sangue mestruale, immondo e sporco).

V. De imbecillitate infantis. (Debolezza del neonato).

a. Nasciamo senza parola, senza scienza, senza virtù: flebili, “imbecilli", poco distanti dagli animali bruti, loro almeno appena nati camminano...

b. "Felici quelli che muoiono prima di nascere, che conoscono la morte prima di conoscere la vita" Geremia $(20,14-18)$ e III cap. di Giobbe.

VI. De dolore partus et eiulatu nascentis. (Del dolore del parto e del pianto del neonato).

a. Tutti nasciamo piangendo per esprimere la nostra miseria;

b. L'uomo entra in questo mondo attraverso il peccato e per il peccato passa la morte a tutti gli uomini.

Miserie della corporeità nell' età adulta (capp. VII-XIII)

VII. De nuditate et vestitu nascentis. (Della nudità dell'uomo).

Citazione di Giobbe che, nato nudo dal ventre della madre, nudo morirà;

VIII. Qualem fructum homo producit. (Quale frutto produce l'uomo) Frutti sporchi che l'uomo, essendo sporco, produce: "tale albero, tale frutto". 
IX. De brevitate huius vite. (Della brevità della vita).

X. De incommodo senectutis. (Degli incomodi della vecchiaia).

Le diverse miserie degli uomini secondo lo stato sociale (capp. XI - XVIII):

Dalla corporeità dell'uomo si passa alle attività dell'uomo, ovviamente tutte vane.

XI. De labore mortalium. (Della fatica dei mortali).

Gli uomini nascono alla fatica, tutti i loro giorni sono pieni di fati che e calamità. Neanche di notte la loro mente riposa.

XII. De diverso studio sapientum. (Le angustie dei sapienti). La ricerca del sapere.

a. Nella molta sapienza, c’è molta indignazione, chi avanza nella scienza accresce il dolore;

b. L'uomo non può spiegare le opere di Dio (Salomone) quindi l'unico modo per trovare la salvezza è Dio.

XIII. De variis hominum studiis. (Dei vari "studi" degli uomini).

Tutte le fatiche e tutte le cose terrene nelle quali gli uomini credo no, diventano vizi vani e afflizione d'animo e nulla di ciò rimane.

XIV. De diversis anxietatibus.

a. Il ricco e il povero, il servo e il signore, il maritato e il celibe, il buono e il cattivo;

b. Tutti, senza distinzione di classe o età, sono afflitti da problemi mondani.

XV. De miseria pauperis et divitis. (Della miseria del povero e del ricco).

a. I poveri vengono disprezzati, la loro condizione è miserabile;

b. $\quad \grave{E}$ meglio morire che essere povero;

c. Ovidio: fin quando sarai felice avrai molti amici, ma quando i tempi si faranno bui (nell'avversa fortuna) ti ritroverai solo;

d. Più l'uomo è ricco più è considerato buono, più è povero più è considerato cattivo;

e. La condizione del ricco diviene miserabile nel momento in cui cade nella trappola dei piaceri e della vana gloria;

f. Conclusione: si fatica nell'acquistare, si teme nel possedere, si ha dolore nel perdere, e questo affatica e affligge la mente. L'uomo dovrebbe fare di Dio il suo tesoro.

XVI. De miseria servorum et dominorum. (Della miseria dei servi e dei signori).

a. La misera ed estrema condizione in cui vive la servitù è la peggiore perché va contro natura: sono nati liberi ma la sorte li ha fatti servi;

b. Orazio conferma che è sempre il popolo che deve scontare gli errori dei governanti e, in senso più generale, sono i subalterni che fanno da capro espiatorio per gli errori dei loro superiori;

c. I servi sono sempre afflitti e non si possono difendere dai signori;

d. Anche la condizione dei signori è misera: se sono crudeli, i servi, 
depravati come sono, lo rispettano e lo temono, se sono clementi sono disprezzati dai suoi sottoposti, che si fanno sfacciati. Il timore, perciò, affligge chi è severo, il disprezzo degrada il mansueto, infatti la crudeltà partorisce l'odio e la confidenza

il disprezzo. Quindi anche i signori sono afflitti giorno e notte.

XVII. De miseria continentis et coniugati. (Della miseria del celibe e del maritato).

XVIII. De miseria bonorum et malorum. (Della miseria dei buoni e dei cattivi).

\section{Altre miserie (capp. XIX-XXIX)}

XIX. De hostibus hominis. (Dei nemici degli uomini che fanno della vita un perpetuo combattimento).

XX. Quod corpus dicitur carcer anime. (Il corpo come carcere dell'anima). La vita è sofferenza, timore e dolore, in nessun luogo si trova la pace, la tranquillità.

XXI. De brevi letitia. (Della breve allegria degli uomini).

XXII. De inopinato dolore. (Del dolore non pensato: le disgrazie impreviste).

XXIII. De vicinitate mortis. (Della vicinanza della morte).

XXIV. De terrore sompniorum. (Dello spavento dei sogni).

a. Causano confusione, fatiche, tormenti;

b. Sporcano l'anima e hanno fatto cadere in errore molti uomini, specialmente chi ha posto in loro la propria speranza;

c. Dove ci sono molti sogni ci sono molte vanità.

XXV. De compassione. (Della compassione).

XXVI. Quod innumere sunt species egritudinum. (Delle innnumerevoli specie di infermità).

XXVII. De subitis infortuniis. (Degli infortuni inaspettati, degli scherzi della fortuna "sfortuna"). Fra le disgrazie inaspettate, le malattie

XXVIII. De diversis generibus tormentorum. (Dei diversi tipi di tormento)

XXIX. De quodam horribili facinore: de quadam muliere que comedit infan tem suum. (Orrendo fatto di una donna che mangiò suo figlio).

\section{Distorsione totale dell'umano giudizio (cap. XXX):}

XXX. Quod quandoque punitur innocens et nocens absolvitur. (Che alcuna fiata si punisce l'innocente, e si libera il reo)

a. L'uomo innocente viene punito, e l'uomo reo assolto;

b. Il pio viene punito e l'empio onorato;

c. Gesù Cristo fu crocifisso e Barabba liberato.

2. Liber secundus: De culpabili humane conditionis progressu (il colpevole sviluppo della condizione umana). Una rassegna dei "beni" (vane apparenze e falsi beni) ai quali di solito gli uomini aspirano nella loro vita terrena (le ricchezze che portano alla malvagità, il sapere che porta al dolore, il potere e gli onori che portano alle vanità, i piaceri che portano alle indecenze) e mostra come la ricerca di questi beni induca inevitabilmente ai vari peccati capitali di cui l'uomo è l'unico artefice e colpevole. 
I. De culpabili humane conditionis progressu. Ci sono principalmente tre cose che gli uomini desiderano nella vita terrena: le ricchezze, il sapere e gli onori.

Dal desiderio delle ricchezze vengono generati due vizi: la Cupidigia e l'Avarizia (capp. II-XVI)

\section{De cupiditate.}

L'avarizia è la radice di tutti i mali e fa commettere agli uomini azio ni scellerate: furti, rapine, guerre, omicidi, inganni.

III. De iniquis muneribus. (Degli iniqui doni).

I doni acciecano gli occhi e che le persone quando li ricevono non giudicano più per "amore iustitie" ma per "amore pecunie".

IV. De acceptione personarum. (Della disparità di trattamento delle persone).

a. Le persone vengono giudicate in base alle loro ricchezze;

b. Vengono distinti $\mathrm{i}$ ricchi dai poveri: i primi agevolati ed ammirati, e secondi disprezzati ed offesi.

V. De venditione iustitie. (Della vendita della giustizia)

a. I ricchi sono corrotti (purrefatti) per amor del denaro e finiscono per imprigionare la loro anima.

VI. De insatiabili desiderio cupidorum. (Dello insaziabile desiderio degli avari).

VII. Quare cupidus satiari non potest. (Perché l'avaro non si può saziare).

a. L'amore per il denaro cresce tanto quanto cresce il denaro;

b. L'avaro non smette mai di desiderare le ricchezze, è insaziabile;

c. Non riceverà mai frutto da quelle.

VIII. De falso nomine divitiarum. (Del falso nome delle ricchezze).

Le ricchezze danno agli uomini una falsa felicità.

IX. Exempla contra cupiditatem. (Esempi contro l'avarizia).

L'avarizia inganna e distrugge.

$\mathrm{X}$. De iniqua possessione divitiarum. (Della ingiusta possessione delle ric chezze).

Molti sono stati distrutti dall'oro e dall'argento.

XI. De licitis opibus. (Delle ricchezze lecite).

a. Chi crede in Dio possiede tutte le ricchezze;

b. È difficile stare nel fuoco e non bruciare, difficile possedere le ricchezze terrene e non amarle.

XII. De incertitudine divitiarum. (Della incertezza delle ricchezze).

Ogni avaro si sforza e combatte contro natura perché la natura con duce l'uomo al mondo povero e alla terra nudo ritornerà.

XIII. De contempnenda possessione divitiarum. (Della superflua sollecitudine degli avari).

Bisogna cercare prima di tutto il regno di Dio ed il resto sarà aggiunto a questo.

XIV. De avaro et cupido. (Il vizio dell'avarizia).

a. L'avaro ha sempre bisogno di denaro anche stando nelle ricchezze; 
b. L'avaro è come l'inferno: riceve e non rende, quindi offende Dio, se stesso e il prossimo.

XV. Cur anaritia dicatur servitus ydolorum. (Perché l'avarizia è servitù degli idoli).

L'avaro serve il tesoro, lo onora, se ne prende cura.

XVI. De proprietatibus avari. (Delle proprietà dell'avaro).

a. Disprezza donare perché ama guadagnare.

b. Porta il suo corpo allo stremo per far crescere il suo guadagno.

c. Le cose ingiuste, le cose che provengono dal male al male pervengono.

d. L'avaro è dannato nella sua vita presente e anche in quella futura.

Dalla ricerca del piacere nascono Gola, Ubriachezza e Lussuria (capp. XVII-XXV) XVII. De gula. (Il vizio della gola).

XVIII. Exempla contra gulam. (Degli esempi contro la gola).

XIX. De ebrietate. (Il vizio della ubriachezza).

XX. Exempla contra ebrietatem. (Degli esempi contro l'ubriachezza).

XXI. De luxuria. (Il vizio della lussuria).

XXII. De generalitate luxurie. (Della generalità della lussuria).

XXIII. De diversis speciebus luxurie. (Delle diverse specie di lussuria e delle pene di quelle).

XXIV. De coitu contra naturam. (Del peccato contro natura).

XXV. De pena huius sceleris. (Della pena di questa scelleratezza).

Dalla ricerca degli onori e del potere nascono Ambizione, Superbia, Arroganza (capp. XXVI-XXXVI)

XXVI. De ambitioso. (Dell'ambizioso).

XXVII. De nimia concupiscentia ambitionis. (Della disordinata concupiscenza degli ambiziosi).

XXVIII. Exemplum de ambitioso. (Esempio dell'ambizioso).

XXIX. Quod brevis est et misera vita magnatum. (Della breve e misera vita dei magnati).

XXX. De diversis proprietatibus superborum. (Delle diverse proprietà dei superbi).

XXXI. De superbia et casu Luciferi. (Della superbia e cadimento di Lucifero).

XXXII. De arrogantia hominum. (Dell'arroganza degli uomini).

XXXIII. De abhominatione superbie. (Dell'abominazione della superbia).

XXXIV. Contra arrogantiam superborum. (Contro l'arroganza dei superbi).

XXXV. Contra fraudem ambitiosorum. (Esempio contro la frode degli ambitiosi).

XXXVI. De proprietatibus arrogantium. (Della proprietà degli arroganti). 
XXXVIII. Contra superfluum ornatum. (Contro il superfluo ornamento).

XXXIX. Quod plus defertur vestibus quam virtutibus. (Perché si onorano più le vesti invece delle virtù).

XL. De ornatu persone, mense et domus.(Degli addobbamenti).

3. Liber tertius: De dampnabili humane conditionis egressu (la condannabile uscita dalla condizione umana) descrive l'apocalisse, il momento della morte e le pene infernali.

Il momento della morte (capp. I-II):

I. De dampnabili humane conditionis egressu.

a. Tutri i beni terreni sono vani perché ogni uomo alla fine della sua vita incontra la morte;

b. Nessuno è puro perché tutti gli uomini peccano;

c. Conclusione: che gli uomini fin quando cercheranno la felicità nei beni terreni non la troveranno mai, anzi riempiranno questo mondo di peccati, esalteranno il male, accumuleranno vizi e moriranno senza potersi salvare.

II. De doloribus quos mali patiuntur in morte. (Dei dolori che i cattivi patiscono nella morte)

a. L'angustia del corpo quando l'anima si stacca dal corpo;

b. "Occhi interiori" nel momento della morte;

c. L'anima viene giudicata e vede per ogni sua iniquità il debito tormento dell'Inferno che dovrà sopportare;

d. L'anima ancora nel corpo vede gli spiriti maligni che si prepararno a prenderla.

Le quattro venute di Cristo sulla terra (cap. III):

III. De adventu Christi ad diem mortis cuiuslibet hominis.

a. La nascita di Cristo sulla terra;

b. Il giorno del giudizio universale quando Cristo tornerà per giudicare tutti gli uomini;

c. Nella mente per grazia divina (Vangelo) nell'anima di ciascun credente nel Battesimo;

d. Nel momento della morte (Giovanni nell'Apocalisse).

Gli orrori della morte corporea e di quella spirituale (capp. IV-XIV)

IV. De putredine cadaverum. (Del fetore dei corpi morti).

V. De tristi memoria dampnatorum. (Della triste memoria dei dannati).

VI. De inutili penitentia reproborum. (Della inutile penitenza dei dannati).

VII. De ineffabili angustia dampnatorum. (Della ineffabile angustia dei dannati).

Delle diverse pene dell'inferno:

VIII. De diversis penis inferni.

a. Le pene infernali sono secondo i diversi peccati: fuoco, freddo, 
fetore, vermi eterni, "flagelli dei percutienti" (Lotario cita Salomone), tenebre esterne e interne, confessione del peccato, orribile visione dei diavoli, catene infuocate.

IX. De igne gehennali. (Del fuoco infernale).

X. De tenebris inferni. (Delle tenebre dell'inferno).

XI. De conversione penarum. (Della confusione delle pene).

XII. De diffidentia dampnatorum. (Della penitenza dei tormenti).

XIII. Cur reprobi numquam liberabuntur a penis. (Che i reprobi mai saranno liberati dalle pene).

XIV. Testimonia de suppliciis eternalibus. (I testimoni dei supplizi eterni).

Il giorno del Giudizio (capp. XV-XX):

$\mathrm{XV}$. De die iudicii.

XVI. De precedente tribulatione. (Della precedente tribolazione).

XVII. Qualiter veniet Dominus ad iudicium.

XVIII. De potentia et sapientia et iustitia indicis. (Della potenza, sapienza e giustizia del giudice).

XIX. De divino iudicio. (Del divino giudizio).

XX. Quod nichil proderit dampnandis. (Che niente gioverà ai dannati).

\section{APPENDICE II}

Schema dell'opera di Bono: Della miseria dell'uomo

1. Miseria dell'uomo $e$ della femmina dall'ora che è creata infino all'uscita del ventre della madre (Capitolo introduttivo: programma della trattazione)

a. nasce nel peccato originale $(I, 1)$

b. viltà della cosa onde è fatta $(I, 2)$

c. viltà della cosa onde si nutrica nel ventre della madre $(I, 3)$

d. dolore della nascita $(I, 4)$

e. miseria della creatura $(I, 5)$

2. Miseria dalla nascita alla morte : dolore-fatica—paura (il programma della trattazione)

a. Dolori: della creatura appena nata (II, 1), nel corso della vita: fisici, psicologici, emotivi (II, 2), nella vechiaia (II, 3).

Rimedi :

1. L'uomo può evitare i mali con la previdenza, o, se non li può evitare, sopportarli con pazienza per "compiacere Dio" (primo bene: II, 4)

2. si rende simile ai santi (secondo bene: II, 5)

3. guadagna il paradiso (terzo bene: II, 6)

b. Fatiche. La vita è fatica; programma della trattazione: fatiche per divenire savio delle cose, per ragunare ricchezze, per i desideri della carne, per le signorie e gli onori. La prima è "vaga e naturale all'uomo e ciascuno vi s'affatica volentieri ", le altre sono fatiche di peccato. 
i. Fatica per divenire savio (III, 1)

ii. Fatica per ragunare ricchezze; programma della trattazione: per divenire ricco d'avere; vanità di queste fatiche; peccati generati dalla ricchezza: l'uomo diventa cupido in accattare ed avaro in ritenere; l'uomo non deve desiderare di fare ricchezze; l'avaro non si sazia; di che cosa l'uomo deve fare tesoro; altre considerazioni; che cosa fa buona la povertà; che cosa fa buona la ricchezza. (III, 2)

1. Fatiche per divenire ricco d'avere (III, 3)

2. Le ricchezze sono false e vane (III, 4)

3. Vizio della cupiditade (III, 5)

4. Vizio dell'avarizia (III, 6). Argomenti contro l'avarizia e perché l'uomo non deve desiderare di fare ricchezze (III, 7); le ragioni per cui l'avaro non si sazia (III, 8); di che cosa l'uomo deve far tesoro e quando lo deve fare (III, 9).

\section{Rimedi}

5. Come mai ci sono stati dei ricchi santi (III, 10)

i. Argomenti per preferire le ricchezze alla povertà (III, 11)

ii. Che cosa fa buona la povertà (III, 12)

iii. Che cosa fa buona la ricchezza (III, 13): Come si devono guadagnare le ricchezze (III, 13); Come si devono spendere e usare (III, 14); Come si devono conservare (III, 15); Altre cose che fanno buona la ricchezza (III, 16); La cortesia, in che modo l'uomo ricco deve essere cortese e come deve usare la cortesia: frenare la lingua, temperare il cuore, spendere e donare le sue ricchezze (III, 17)

iv. Perché la vita povera viene considerata dai Santi come beata, perfetta e migliore di quella ricca (III, 18)

iii. Fatiche per i desideri della carne (III, 19)

1. Vizio della gola ed il male che ne segue (III, 19)

2. Vizio della lussuria ed il male che ne segue (III, 20)

3. I rimedi contro la lussuria (III, 21)

iv. Fatiche delle signorie e degli onori (III, 22)

4. Vizio della superbia (III, 23)

5. Vizio della vanagloria (III, 24)

c. paura (programma della trattazione)

i. quattro paure: demonio, carne, uomo, natura; sogni (IV, 1)

ii. I rimedi $(\mathrm{IV}, 2)$

3. Morte e miseria dopo la morte (programma della trattazione)

a. va in inferno colui che non osserva i comandamenti di Dio $(\mathrm{V}, 1)$.

Quali sono i comandamenti $(\mathrm{VI}, 1)$

i. i maggiori comandamenti (VI, 2): amore di Dio (VI, 3), amore del prossimo (VI, 4).

ii. i tre comandamenti minori che riguardano l'amore di Dio (VI, 5)

iii. i cinque comandamenti minori che riguardano l'amore del 
prossimo (VI, 6)

b. sorte dell'anima dopo la morte (VI, 7)

c. l'inferno e i suoi tormenti (VI, 8-9): il tormento dei pensieri (VI, 10); argomento della non eternità dell'inferno (VI, 11); contestazione del precedente (VI, 12)

4. Beatitudine e gloria del giusto che va in Paradiso (VII, 1)

a. come è disposto il Paradiso

b. la beatitudine e la gloria delle anime che vanno in Paradiso (VII, 2)

c. delle potenze dell'anima: immaginare, lavorare, desiderare (VII, 3)

d. la potenza che c'è nell'anima nel lavorare e perché vivendo ci si affati-

ca senza trovare riposo e come ci si riposa invece in Paradiso (VII, 4)

e. la potenza che c'è nell'anima nel desiderare e come nel mondo non si può saziare e come si sazia in Paradiso (VII, 5)

5. Il giorno del Giudizio: le cose che avvengono prima; i segnali; il giorno del giudizio; la sentenza che dà Dio (VIII, 1)

i. in che modo nel giorno del Giudizio finirà il mondo intero (VIII, 2)

ii. i quindici segni del Giudizio (VIII, 3)

iii. in che modo nel giorno del Giudizio resusciteranno e saranno giudicate le anime (VIII, 4)

\section{OPERE CITATE}

Barberi Squarotti, Giorgio. Storia della civiltà letteraria italiana, 7 voll. Torino: UTET, 1990.

Debenedetti, Santorre. Bono Giamboni, "Studi Medievali", 4 (1912-13): 271-278.

Divizia, Paolo. Ancora un compendio del Libro de' Vizi e delle Virtudi di Bono Giamboni, Medioevo Romanzo, 27:1 (2003): 33-43.

—. I quindici segni del Giudizio: appunti sulla tradizione indiretta della Leggenda aurea nella Firenze del Trecento, in Studi su volgarizzamenti due-trecenteschi, a cura di P. Rinoldi e G. Ronchi, Roma, Viella, 2004, pp. 1-21.

Foà, Simona. Bono Giamboni, in Dizionario biografico degli italiani. Roma, Istituto della Enciclopedia Italiana, 2000, vol. 54 ad vocem.

Giamboni, Bono. Della miseria dell'uomo. Giardino di consolazione. Introduzione alle virtú, di Bono Giamboni, aggiuntavi La scala dei claustrali, testi inediti, tranne il terzo trattato, a cura di Francesco Tassi, Firenze: Guglielmo Piatti, 1836.

Fiore di rettorica, a cura di Gian Battista Speroni. Pavia: Dipartimento di Scienza della Letteratura e dell'Arte medioevale e moderna, 1994.

Il libro de' Vizi e delle Virtudi e il Trattato di virtù e di vizi, a cura di Cesare Segre. Torino: Giulio Einaudi editore, 1968.

Lotario de' Segni (Papa Innocenzo III). De miseria conditionis humane, a cura di Robert E. Lewis. Athens: The University of Georgia Press, 1978.

Lotharii Cardinalis (Papa Innocenzo III). De miseria humane conditionis, a cura di 
Michele Maccarrone. Lucani: in aedibus Thesauri Mundi, 1955.

Mazzon, A. "Il Libro de' Vizi e delle Virtudi di Bono Giamboni. Un nuovo testimone presso la Biblioteca Civica di Padova, Critica del Testo, 4:2 (2001): 461467.

Ruggiero, Romano - Vivanti, Corrado. Storia d'Italia. Vol. 2. Torino: Giulio Einaudi, 1974.

Segre, Cesare. Bono Giamboni, in Dizionario critico della letteratura italiana, a cura di Vittore Branca. Torino, UTET, 1986, vol. 1, ad vocem.

Segre, Cesare - Marti, Mario (a cura di), La prosa del Duecento, Milano-Napoli: Ricciardi, 1959, pp. 227-254 e 1063-64. (Contiene una edizione semi-critica dei trattati VI e VII del Della miseria dell'uomo). 